\title{
Bases cartográficas para armazenamento e análise espacial de dados da diversidade de palmeiras em um trecho de Mata Atlântica, Ubatuba-SP
}

\author{
Maria Cecília Barbosa de Toledo ${ }^{1} \&$ Simey Thury Vieira Fisch ${ }^{2}$
}

Biota Neotropica v6 (n1) - http://www.biotaneotropica.org.br/v6n1/pt/abstract?article+bn01806012006

Recebido em 18/05/2005.

Versão revisada recebida em 20/12/2006.

Publicado em 01/01/2006

\author{
${ }^{1}$ Departamento de Biologia/Universidade de Taubaté - ceciliabt@vivax.com.br \\ ${ }^{2}$ Departamento de Biologia/Universidade de Taubaté - simey@unitau.br \\ Endereço para correspondência: \\ Universidade de Taubaté - Departamento de Biologia \\ Grupo ecossistemas terrestres do Vale do Paraíba e Litoral Norte paulista \\ Praça Marcelino Monteiro, 63 -Taubaté - SP - 12030-010 - Brasil
}

\begin{abstract}
Toledo, M.C.B. and Fish, S.T.V. Cartographic basis for storage and spatial analysis of data on diversity of palm trees in a stretch of Mata Atlântica, Ubatuba - SP. Biota Neotrop. Jan/Abr 2006 vol. 6, no. 1,http://www.biotaneotropica.org.br/ v6n1/pt/abstract?article+bn01806012006. ISSN 1676-0603

The aim of this work was to elaborate a cartographic basis for storage and spatial analysis of data on diversity of palm trees in altitudinal gradient. The work was carried out in Serra do Mar, located on the northeast of São Paulo. In order to create a databases an altimetric letter from IBGE 1:50.000 and a Landsat ETM+ image from 2002 in bands 3,4 and 5 have been used. Data have been analyzed in the SPRING System of Geographic Information 3.6 version, developed by the National Institute for Space Research (INPE), Brazil. The sequence of elaboration consisted of register, digitalization of level curves, and creation of a rectangular, triangular and data table. From this sequence declivity and hypsometric thematic maps have been obtained. Later on the association of the number of species by altitudinal class giving origin to a map of palm tree species distribution in an altitudinal gradient was carried out. The databases are available for association to other parameters with climate, soil, distribution, and abundance of palm tree species for more elaborated spatial analysis.
\end{abstract}

Key words: thematic maps, altitudinal gradient, diversity of Arecaceae, GIS

\section{Resumo}

Toledo, M.C.B. and Fish, S.T.V. Bases cartográficas para armazenamento e análise espacial de dados da diversidade de palmeiras em um trecho de Mata Atlântica, Ubatuba-SP. Biota Neotrop. Jan/Abr 2006 vol. 6, no. 1 http:// www.biotaneotropica.org.br/v6n1/pt/abstract?article+bn01806012006. ISSN 1676-0603

O objetivo desse trabalho foi a elaboração de uma base cartográfica para armazenamento e análise espacial dos dados de diversidade de palmeiras em um gradiente altitudinal. O trabalho foi realizado na Serra do Mar na região nordeste do Estado de São Paulo. Para montagem do banco de dados utilizou-se uma carta altimétrica do IBGE 1:50.000 e uma imagem Landsat ETM+ de 2002 nas bandas 3,4 e 5. Os dados foram analisados no Sistema de Informação Geográfica SPRING versão 3.6 desenvolvido pelo Instituto Nacional de Pesquisas Espaciais. A seqüência de elaboração consistiu de registro, digitalização das curvas de nível, criação da grade retangular, grade triangular e fatiamento. Dessa seqüência obteve-se mapas temáticos de declividade e hipsométrico. Esse último foi vetorizado e poligonizado. Posteriormente foi realizada a associação do número de espécies de palmeiras por classe altitudinal, originando um mapa de distribuição de espécies por gradiente altitudinal. O banco de dados encontra-se disponível para associar outros parâmetros como clima, solo, distribuição e abundância das espécies de palmeiras, objetivando análises espaciais mais elaboradas.

Palavras-chave: mapas temáticos, gradiente altitudinal, diversidade de Arecaceae, SIG 


\section{Introdução}

O avanço tecnológico na representação do espaço possibilitou não somente a ampliação do uso de informações cartográficas já existentes (cartas planialtimétricas e levantamentos aerofotográficos) como também a intergração dessas com novas tecnologias tais como os sistemas de posicionamento global - GPS. Atualmente, sensores sofisticados sejam aerotransportados ou satelitais utilizados para a obtenção de imagens, scanners de alta resolução que permitem a transformação de cartas em papel em formato digital aumentam a quantidade de dados analisados, de forma a permitir um melhor entendimento dos processos naturais e antrópicos em escalas mais amplas. Os Sistemas de Informação Geográfica (SIGs) permitem realizar análises espaciais, criar uma relação topológica entre os elementos gráficos além de permitir uma descrição de suas entidades: pontos, linhas e polígonos (Rodrigues, 1990). Dessa forma, os SIGs possibilitam a associação dos dados espaciais com atributos observados em campo, como por exemplo, dados ecológicos.

A utilização de técnicas de análise de dados distribuídos espacialmente por meio de SIGs integrado a ecologia é relativamente recente e permite um melhor entendimento das inter-relações dos diferentes elementos físicos e biológicos. Segundo Metzger (2001), foi a partir da década de 80 que os ecólogos vêm ampliando o uso dessa integração, porém a complexidade da ferramenta e a demanda de tempo para assimilar as novas técnicas de georreferenciamento e sensoriamento remoto muitas vezes inviabilizam sua utilização mais freqüente. O interesse na análise espacial e temporal é o de obter respostas para questões ecológicas, utilizando escalas mais amplas. Johnston (1998) refere-se a algumas dessas perguntas: (1) Como está distribuída a comunidade A? (2) Como está distribuída a comunidade A em relação à comunidade B? (3) Como está distribuída a espécie A em relação aos fatores ambientais X,Y e Z? (4) Como tem mudado a distribuição de plantas da comunidade A no tempo passado? (5) Como será a distribuição da comunidade de plantas A no futuro, se as condições ambientais continuarem as mesmas? (6) Como será a distribuição de plantas da comunidade A se o fator ambiental $\mathrm{X}$ for alterado? Em médio prazo e com trabalhos de campo aliados a informações obtidas em bases cartográficas, estas questões poderão ser respondidas.

Uma base de dados georreferenciada é fundamental para analisar a distribuição espacial de vegetais ou animais. A partir da consulta a um banco de dados espaciais informações como localização, altitude e declividade são resultados imediatos que podem ser associados a dados de ocorrência e abundância de uma determinada espécie.

É na América do Sul onde a família Arecaceae, tipicamente pantropical, ocorre em hábitats diversos e em diferentes altitudes (Moore 1973). Na floresta atlântica, que se estende ao longo da costa brasileira, a presença da Serra do Mar permite um ambiente úmido ocasionado pela brisa marítima e contra-forte montano, sendo comum em suas encostas a presença de palmeiras dos gêneros Euterpe, Bactris, Geonoma e Attalea (Henderson et al. 1995).

Dessa forma, o objetivo do presente trabalho foi descrever e verificar a viabilidade do uso e interpretação de dados georreferenciados e espacialmente distribuídos por meio da construção de uma base de dados e do mapeamento de variáveis discretas como declividade, altitude e número de espécies de palmeiras.

\section{Material e método}

\section{Área de estudo:}

A área compreende a bacia do rio Grande de Ubatuba, região no domínio de Mata Atlântica, localizada na escarpa da Serra do Mar entre as coordenadas 45W 11'22"; $23 \mathrm{~S}$ 28’02" e 45W 03’55"; 23S 20’44". O clima dessa região é tropical chuvoso com temperaturas altas, e muito úmido no verão. Nessa área uma parcela significativa das chuvas é oriunda de convecção topográfica (Fisch 2003).

As regiões da faixa da Serra do Mar e bacia do Paraíba do sul apresentam uma variação altitudinal de 0 a 1.300 metros acima do nível do mar, estando sujeitas aos mais fortes processos de erosão e de movimentos coletivos de solo. A área caracteriza-se pela ocorrência de decomposição das rochas cristalinas ou cristalofilianas de 3 a $60 \mathrm{~m}$ de profundidade; pela presença de solos tipo latossolo, pela freqüente presença de solos superpostos, ou seja, coberturas coluviais soterrando stone lines e por precipitações que variam entre 1100 e 1500 mm ao ano. Quanto à unidade morfoclimática e climato-botânicas, caracterizamse como regiões serranas, de morros mamelonares do Brasil Sudeste (Ab’Sáber 2003).

A vegetação na área de estudo classifica-se como Floresta Ombrófila Densa, também denominada de Mata Atlântica (Silva \& Leitão-Filho 1982, Veloso et al. 1991, Simonetti 2001). A família Arecaceae, dentre outras, é uma das famílias mais importantes na composição florística e estrutural desse trecho de Mata Atlântica, no município de Ubatuba. Além desta família, é característica a representatividade de famílias de dispersão universal, com um número de espécies significativas de Myrtaceae, Rubiaceae, Lauraceae e Melastomataceae (Silva \& LeitãoFilho 1982, Lacerda 2001).

\section{Amostragem das palmeiras:}

Para o estudo da distribuição da comunidade de palmeiras no gradiente altitudinal, as espécies foram amostradas nas cotas 0 (2m do nível do mar) 100, 200, 400, 600 e 850 m na trilha da vargem grande (Tabela 1). Em função da ocupação humana, no caso da cota de $0 \mathrm{~m}$ de altitude a amostragem na foi realizada em outra área (restinga da 
Picinguaba). Em cada altitude, foi estendida uma linha de $200 \mathrm{~m}$ acompanhando a superfície do terreno, ao longo da qual foram sorteados 10 pontos de onde partiram transectos com 50 m de comprimento, perpendiculares aos $200 \mathrm{~m}$. Nos transectos foram sorteados 25 pontos nos quais foram alocados os centros das parcelas circulares de $100 \mathrm{~m}^{2}$ (adaptado de Gentry 1990), onde todas as palmeiras, plântulas e adultos, tiveram sua presença registrada.

\section{Elaboração do banco de dados:}

Para o geoprocessamento foi utilizado o Sistema de informação Geográfica SPRING 3.6-(INPE 2000) e disponível gratuitamente na rede mundial. Utilizou-se uma carta planialtimétrica 1:50.000 da região de Ubatuba fornecida pelo IBGE, a qual foi transformada em imagem matricial e importada para o ambiente SPRING. A partir da entrada da carta no SIG, iniciou-se o trabalho de digitalização dos dados. Também foi criado um plano de informação para entrada da imagem Landsat ETM+ do ano de 2002 nos canais TM3, TM4 e TM5 para elaboração da imagem tridimensional, segundo esquema apresentado na Figura 2.

\section{Resultados e Discussão}

\section{Espécies de palmeiras}

Nove espécies de palmeiras foram amostradas (Euterpe edulis, Syagrus peseudococos, Attalea dubia, Bactris setosa, B. hatschbachii, Astrocaryum aculeatissimum, Geonoma gamiova, G. pohliana, G. elegans) ao longo da encosta montana. O maior número de espécies (n=8) foi observado nas altitudes 100 e 200m, e o menor número $(n=4)$ foi obtido na cota $850 m$ (Tabela 2).

\section{Banco de dados}

A partir da digitalização das curvas de nível da carta Ubatuba do IBGE foi obtido um plano de informação associado à categoria de Modelo Numérico de Terreno, como mostra a Figura 3. Os mapas topográficos geralmente representam dados de elevação dentro de um intervalo e este intervalo é representado por isolinhas, sendo perfeitamente aceitável para representar características tridimensionais sobre uma superfície bidimensional fornecendo um bom entendimento do relevo (Johnston, 1998).

Para a representação tridimensional é necessária a criação de um Modelo Numérico do Terreno. Este pode ser uma grade retangular (matriz de altitude) e/ou uma grade triangular (TIN - Triangular Irregular Networks) (Burrough \& McDonnell, 1998). A grade retangular, no modelo raster, foi obtida utilizando o vizinho mais próximo como interpolador e posteriormente uma grade triangular usando o interpolador linear, Figuras 4 e 5, respectivamente.
Através da imagem Landsat registrada, de acordo com a carta do IBGE, e realçada obteve-se uma imagem sintética. A partir da sobreposição da imagem à grade retangular foi gerada a imagem 3D. A imagem em três dimensões que permite que 0 relevo da área de trabalho seja observado de acordo com a realidade de campo, está apresentada na Figura 1.

Para a criação do mapa altitudinal foi feito o fatiamento a partir da cota mínima (0 m) até a cota máxima (1200m) com um intervalo de $100 \mathrm{~m}$. Para cada intervalo de 100 metros foi atribuída uma classe, como segue a legenda da Figura 6. Observou-se que nas faixas mais estreitas a altitude atinge de 500 a $900 \mathrm{~m}$ em um pequeno trecho no terreno e o platô conservou-se entre 800 a 1100m, com alguns picos chegando a $1200 \mathrm{~m}$ de altitude.

O mapeamento da declividade da área de estudo foi obtido a partir da grade triangular, com o dado de saída da declividade em percentagem em um modelo temático de representação. Para tanto, foram definidos 8 intervalos de classes de 0 a $80 \%$ de declividade. As áreas mais declivosas (80\%) foram detectadas na crista da serra onde pôde ser observado a existência de uma linha de afloramento rochoso (Figura 7). Esta área não foi avaliada quanto à ocorrência de palmeiras pela inviabilidade de acesso.

Quanto ao mapa hipsométrico foi criado um plano de informação vetorizado e poligonizado, criando-se classes de número de espécies por faixa de $100 \mathrm{~m}$ de altitude, num total de 12 classes, indo de 0 até $1200 \mathrm{~m}$ de altitude. A distribuição das espécies foi obtida a pela sobreposição do número de espécies (Tabela 2) e o mapa hipsométrico (Figura 6). A figura 8 apresenta a distribuição das espécies segundo a sua localização geográfica e o nível altitudinal de ocorrência.

Segundo Burrough \& McDonnell (1998) um dos usos mais comuns dos modelos de elevação é como base para visualização de informações temáticas ou para combinar dados de relevo com dados temáticos como solo, uso do terreno ou vegetação. Os dados obtidos em campo foram utilizados para definir as classes de ocorrência do número de espécies de palmeiras em cada faixa de 100 m de altitude.

Toledo (2003) realizou uma análise de entidades discretas no espaço como a diversidade biológica por meio da criação de um banco de dados contendo informações de variáveis como abundância de espécie, distribuição, freqüência, índice de diversidade, entre outras tanto da fauna como da flora. Pivello \& Bitencourt (1999) também construíram um banco de dados para uma área de cerrado, utilizando dados do ambiente físico e biológico com o objetivo de zoneamento e manejo de uma área de conservação por meio da elaboração de um plano de informação cadastral em que os polígonos, representantes de classes distintas, foram associados a atributos como estádios florestais, solo e relevo, entre outros, que foram integrados a uma matriz de variáveis. 
A partir da construção desta base cartográfica será possível a elaboração de uma tabela de dados com uma maior representatividade de parâmetros físicos como declividade, clima e solo, e biológicos como distribuição e abundância das espécies de palmeiras em toda a microbacia, permitindo realizar não só mapeamentos temáticos como também uma análise espacial desses parâmetros.

\section{Agradecimentos}

À Dra Silvia María Lacruz pelas sugestões no manuscrito. A equipe de coleta de dados de palmeira do projeto "Distribuição a comunidade de palmeiras do gradiente altitudinal da floresta atlântica na região nordeste do Estado de São Paulo” (proc. 01/06023-5). Ao Programa BIOTA/FAPESP pelos recursos financeiros que permitiram a realização dos trabalhos.

\section{Referências Bibliográficas:}

AB'SABER, A. 2003. Os domínios de natureza no Brasil. Potencialidades paisagísticas. Ateliê Editorial. São Paulo. 159pp.

BURROUGH, P.A.; McDONNELL, R.A. 1998. Principles of geographic information systems. Oxford University Press, New York. .

FISCH, G. 2003. Distribuição da comunidade de palmeiras no gradiente altitudinal da Floresta Atlântica na região nordeste do estado de São Paulo. Resumo In IV Simpósio e IV Reunião de Avaliação do Programa Biota/Fapesp. Águas de Lindóia.

GENTRY, A.H. 1990. Floristic similarities and differences between Southern Central America and upper and Central Amazonia. In: Four neotropical rain forest. Ed. Yale University Press, London, p. 141-160.

HENDERSON, A.; GALEANO, G.; BERNAL, R. 1995. Field Guide to the Palms of the Americas. New Jersey, Princeton University Press, 352 p.

INPE. 2000. Manual do SPRING. Versão 5.3.1. São José dos Campos, .

JOHNSTON, C.A. 1998. Geografic information systems in ecology - methods in ecology series. Blackwell Science Ltd. Oxford, UK. 239 p.

LACERDA, M. S. 2001. Composição florística e estrutura da comunidade arbórea num gradiente altitudinal da Mata Atlântica. Tese de Doutorado. Campinas, SP.Referências Bibliográficas

METZGER, J.P. 2001. O que é ecologia de paisagens? Biota Neotropica 1/2 http://www.biotaneotropica.org.br/v1n12/ pt/fullpaper?bn00701122001+pt
MOORE, H.E. 1973. Palms in the tropical forest ecosystems in Africa and South America. In: MEGGERS, B.J.; AYENSY, E.S.; DUCKWORTH, W.D. (eds.). Tropical forest ecosystems in Africa and South America: a comparative review. Washington DC, Smithsonian Institution Press, p. 63-88.

PIVELLO, V.R. \& BITENCOURT, M.D. 1999. Banco de dados em SIG para ecologia aplicada: exemplo do cerrado Péde-Gigante, SP. Caderno de Informações Georreferenciadas - CIG. 1(3): disponível em www.cpa.unicamp.br/revista/cigv1n3a4.html. Acessado em 13 de dezembro de 2004.

RODRIGUES, M. 1990. Introdução ao geoprocessamento. In: SIMPOSIO BRASILEIRO DE GEOPROCESSAMENTO, 1, São Paulo. Anais. São Paulo: EDUSP, v.1, p.1-26.

SILVA, A.F. \& LEITÃO FILHO, H.F. 1982. Composição florística e estrutura de um trecho de mata atlântica de encosta no município de Ubatuba (São Paulo, Brasil). Rev. bras. Bot. 5(1):43-52.

SIMONETTI, C. 2001. As relações entre o relevo, os solos e a Floresta Atlântica na Serra do Mar (Bacia do rio Itamambuca, Ubatuba, SP). Tese de doutorado. Universidade de São Paulo, SP.

TOLEDO, M.C.B., 2003. Comunidade de aves como bioindicadoras para diagnóstico e avaliação da diversidade em uma paisagem urbana. Tese de Doutorado. Universidade de Taubaté, São Paulo, SP.

VELOSO, H. P.; RANGEL FILHO, A. L. R.; LIMA, J. C. A. 1991. Classificação da vegetação brasileira, adaptada a um sistema universal. IBGE, Rio de Janeiro.

Título: Bases cartográficas para armazenamento e análise espacial de dados da diversidade de palmeiras em um trecho de Mata Atlântica, Ubatuba-SP

Autores: Maria Cecília Barbosa de Toledo \& Simey Thury Vieira Fisch

Biota Neotropica, Vol. 6 ( número 1): 2006

http://www.biotaneotropica.org.br/v6n1/pt/ abstract?article+bn01806012006

Recebido em 18/05/2005 - Revisado em 20/12/2006

Publicado em 01/01/2006

ISSN 1676-0603 


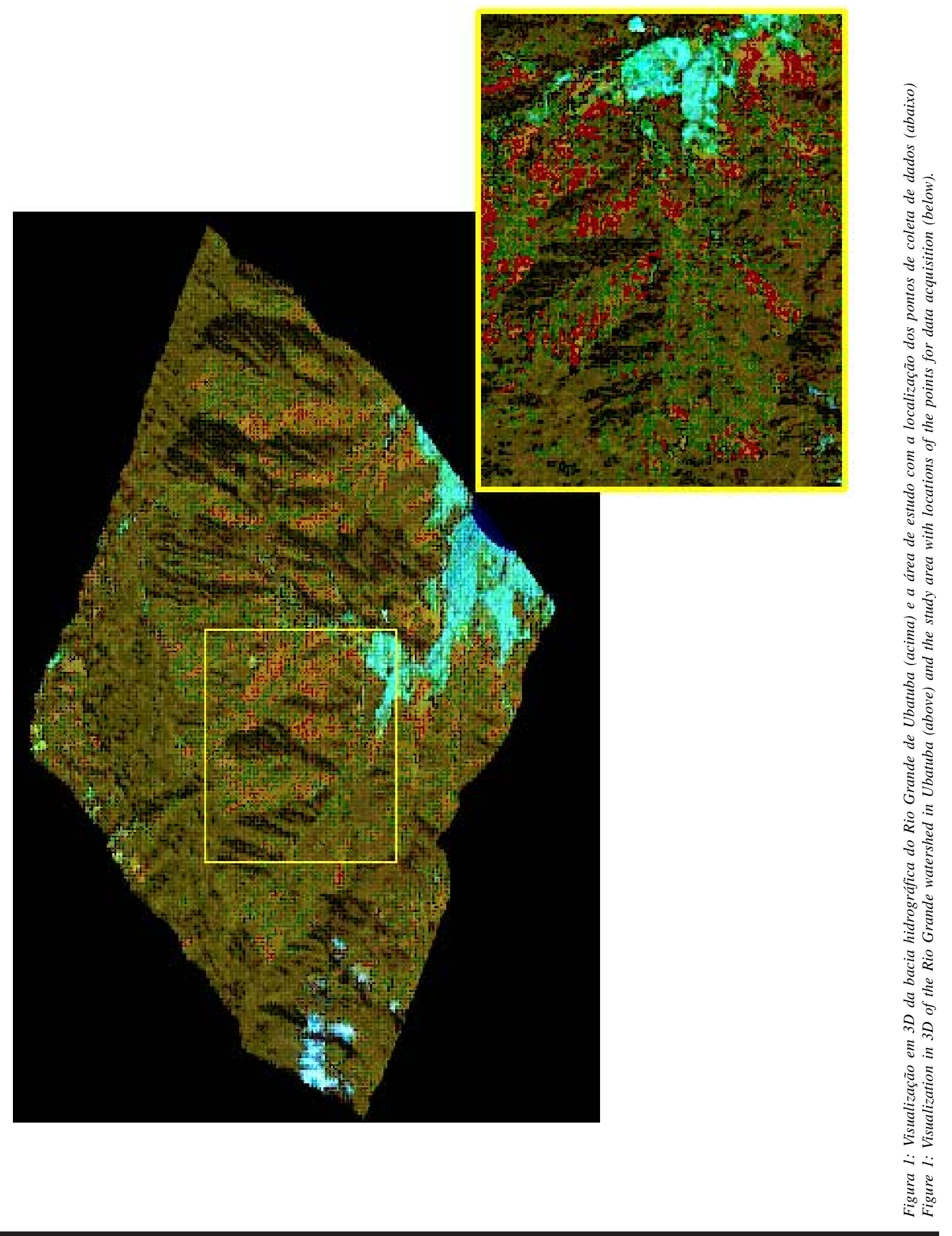

http://www.biotaneotropica.org.br 


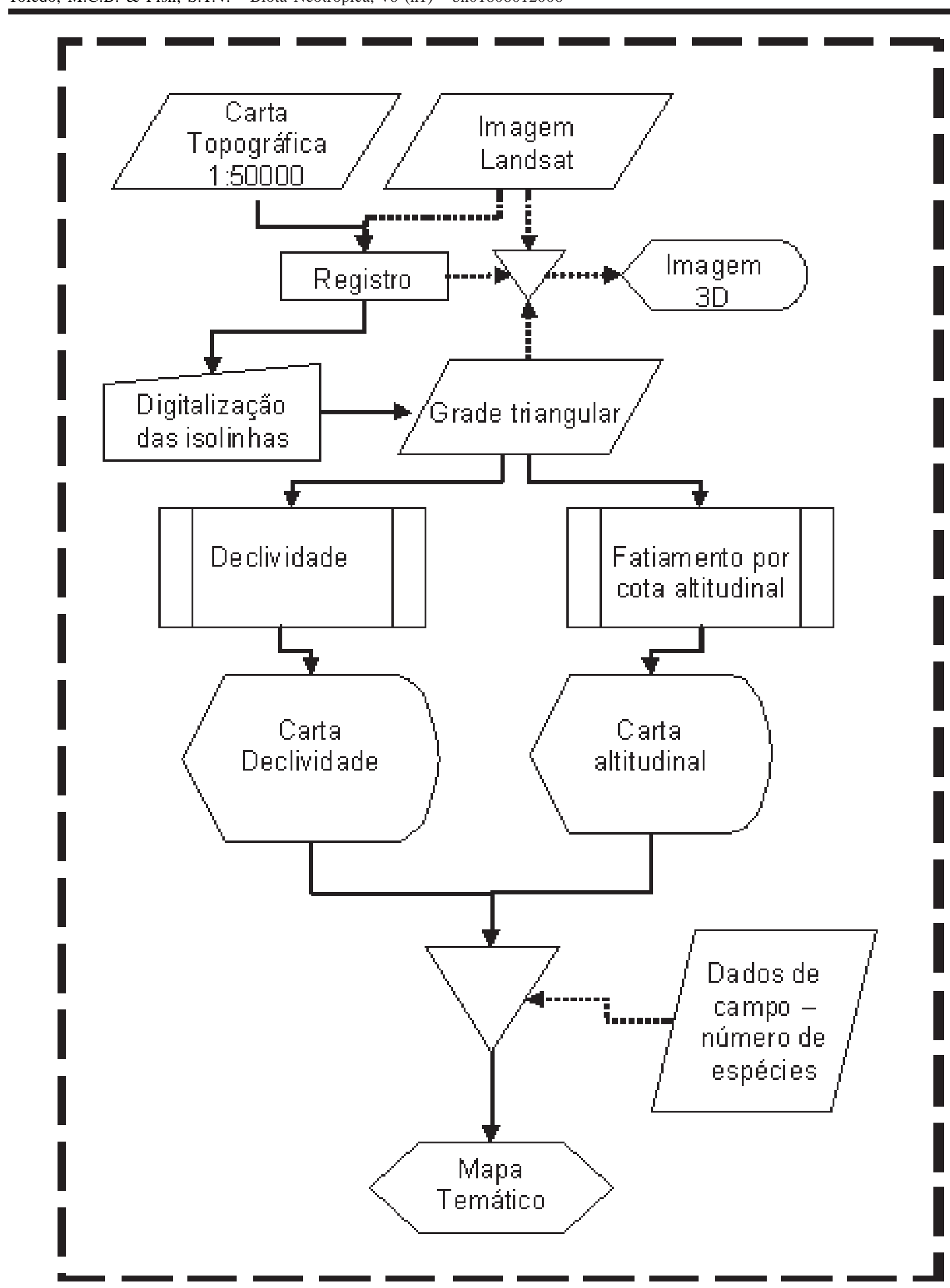

Figura 2: Fluxograma da metodologia utilizada para construção mapa de distribuição altitudinal do número de espécies de palmeiras. Figure 2: Methodology flowchart used for building altitudinal maps of palms species distribution. 
Toledo, M.C.B. \& Fish, S.T.V. - Biota Neotropica, v6 (n1) - bn01806012006


Figura 3: Mapa topográfico digitalizado a partir da carta do IBGE. Em azul as isolinhas e os sinais de soma em vermelho correspondem aos pontos de controle.

Figure 3: Topographic map generated from the digitalized IBGE/Ubatuba chart. The blue isolines and the red plus signs are related to control points. 
Toledo, M.C.B. \& Fish, S.T.V. - Biota Neotropica, v6 (n1) - bn01806012006

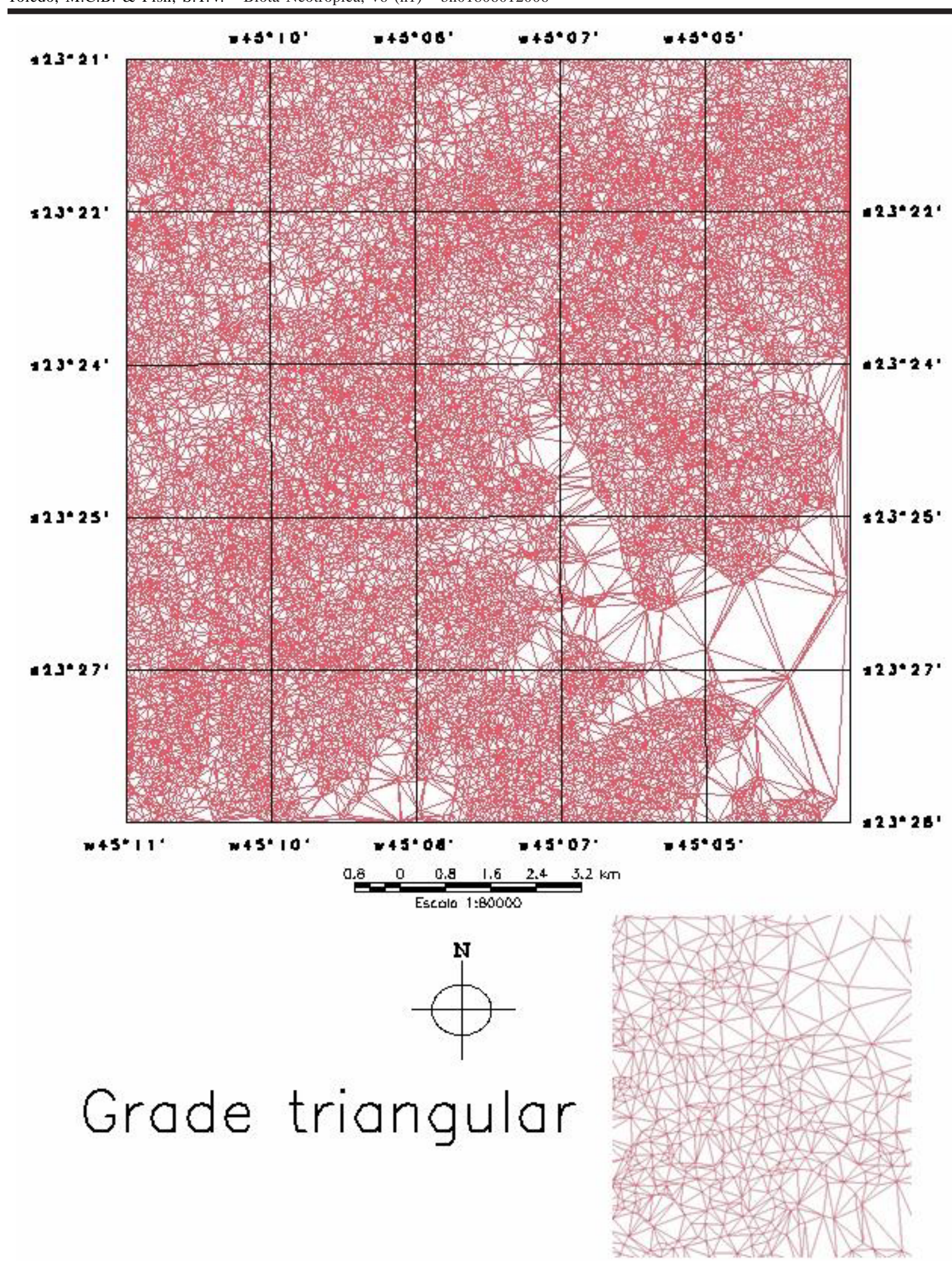

Figura 4: Grade triangular gerada a partir da digitalização da carta de Ubatuba do IBGE.

Figure 4: Triangular grade generated from the digitalized IBGE/Ubatuba chart. 


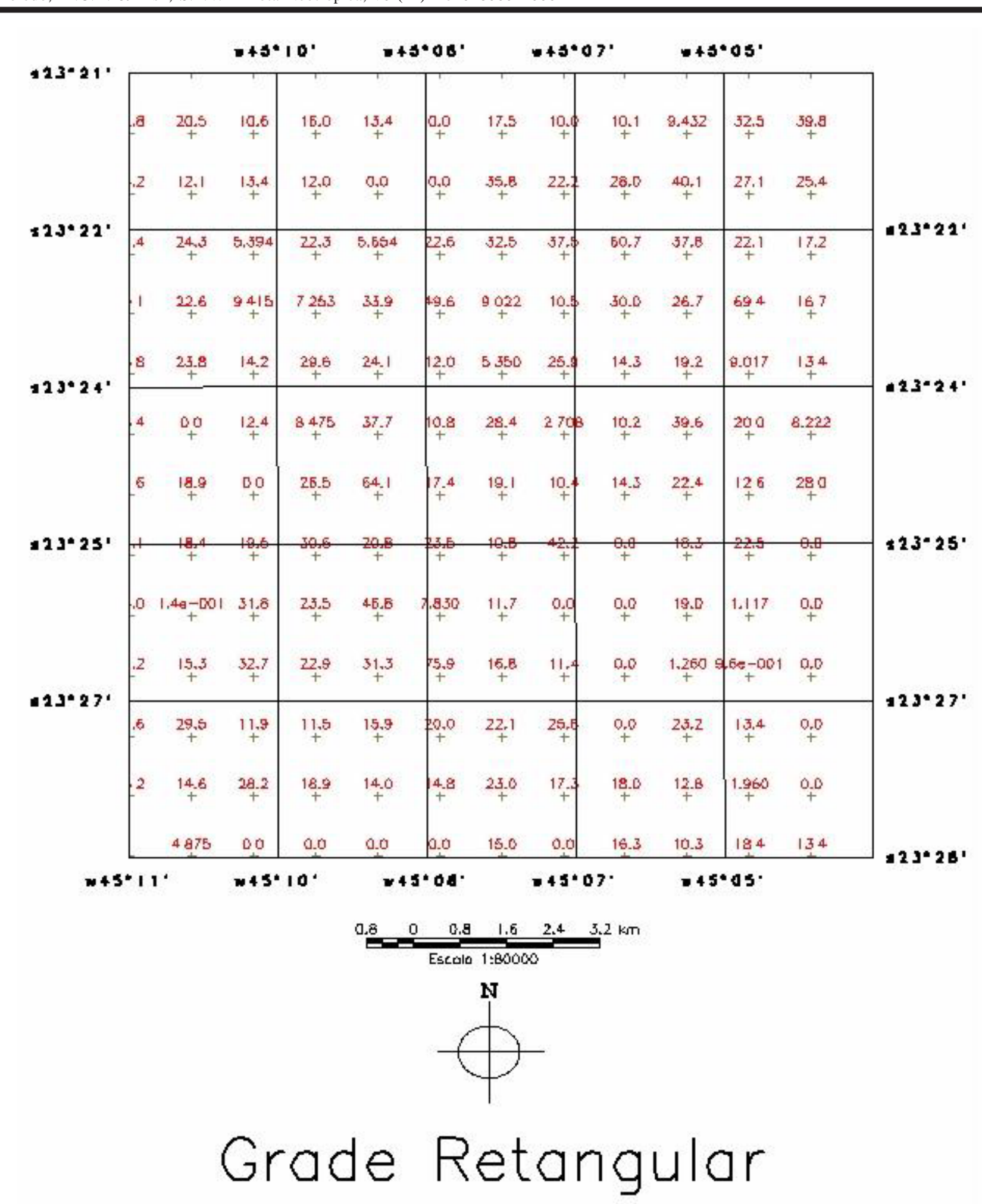

Figura 5: Grade retangular gerada a partir da digitalização da carta Ubatuba do IBGE

Figure 5: Rectangular grade generated from the digitalized IBGE/Ubatuba chart. 


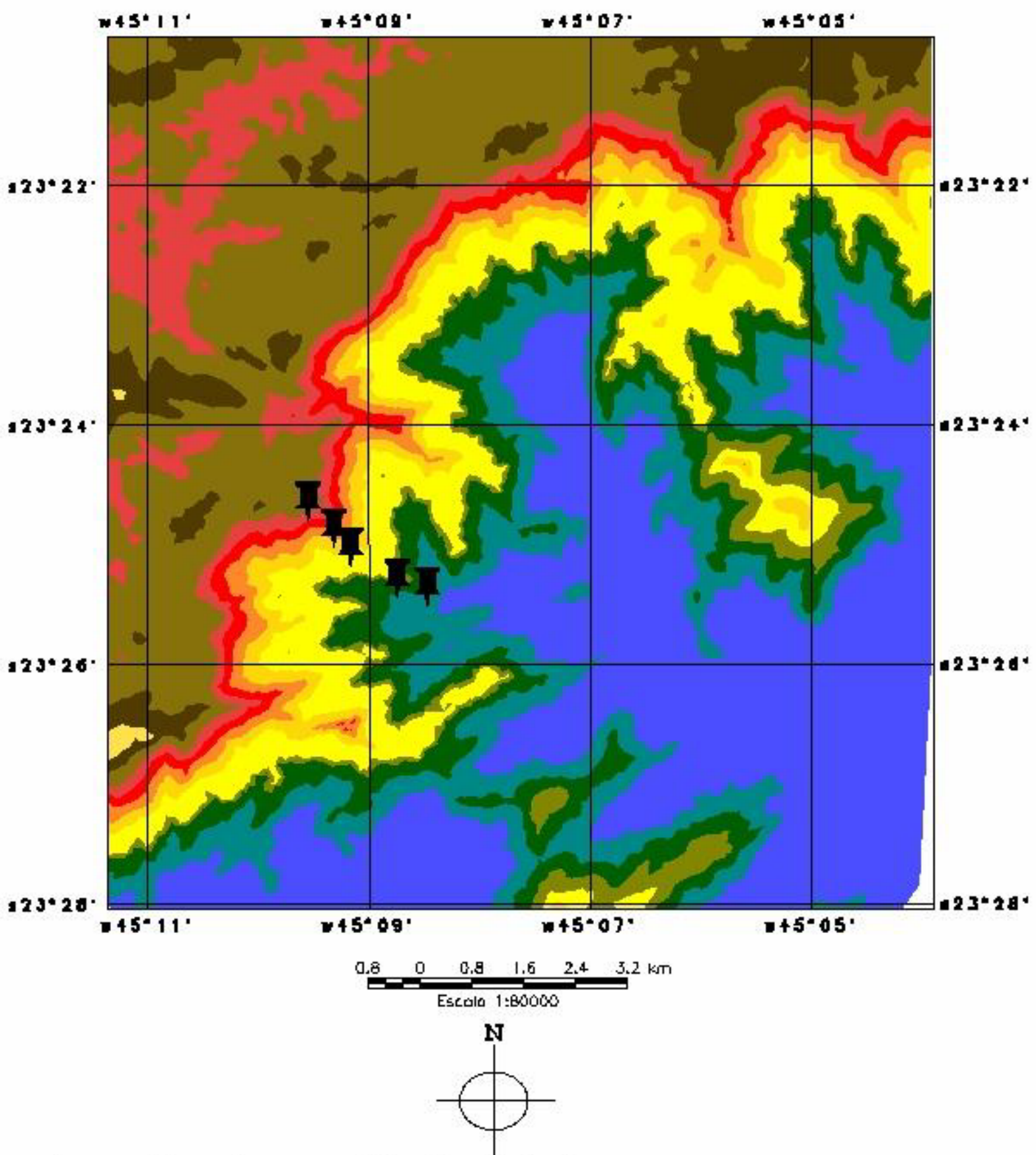

Legenda: classes altitudinais ( $m$ )

$\begin{array}{lll}0-100 & 600-700 & \text { FPontos amostrais } \\ 100-200 & 700-800 \\ 200-300 & 800-900 \\ 300-400 & 900-1000 \\ 400-500 & 1000-1100 \\ 500-600 & 1100-1200\end{array}$






Legenda: declividade (\%)

$\begin{array}{lll}0-10 & 40-50 \\ 10-20 & 50-60 \\ 20-30 & 60-70 \\ 30-40 & 70-80\end{array} \quad$ TPontos amostrais

Figura 7: Mapa de declividade da área de estudo indo de 0 a 80\% de inclinação.

Figure 7: Declivity maps of the study area going from 0 to $80 \%$ of slope. 


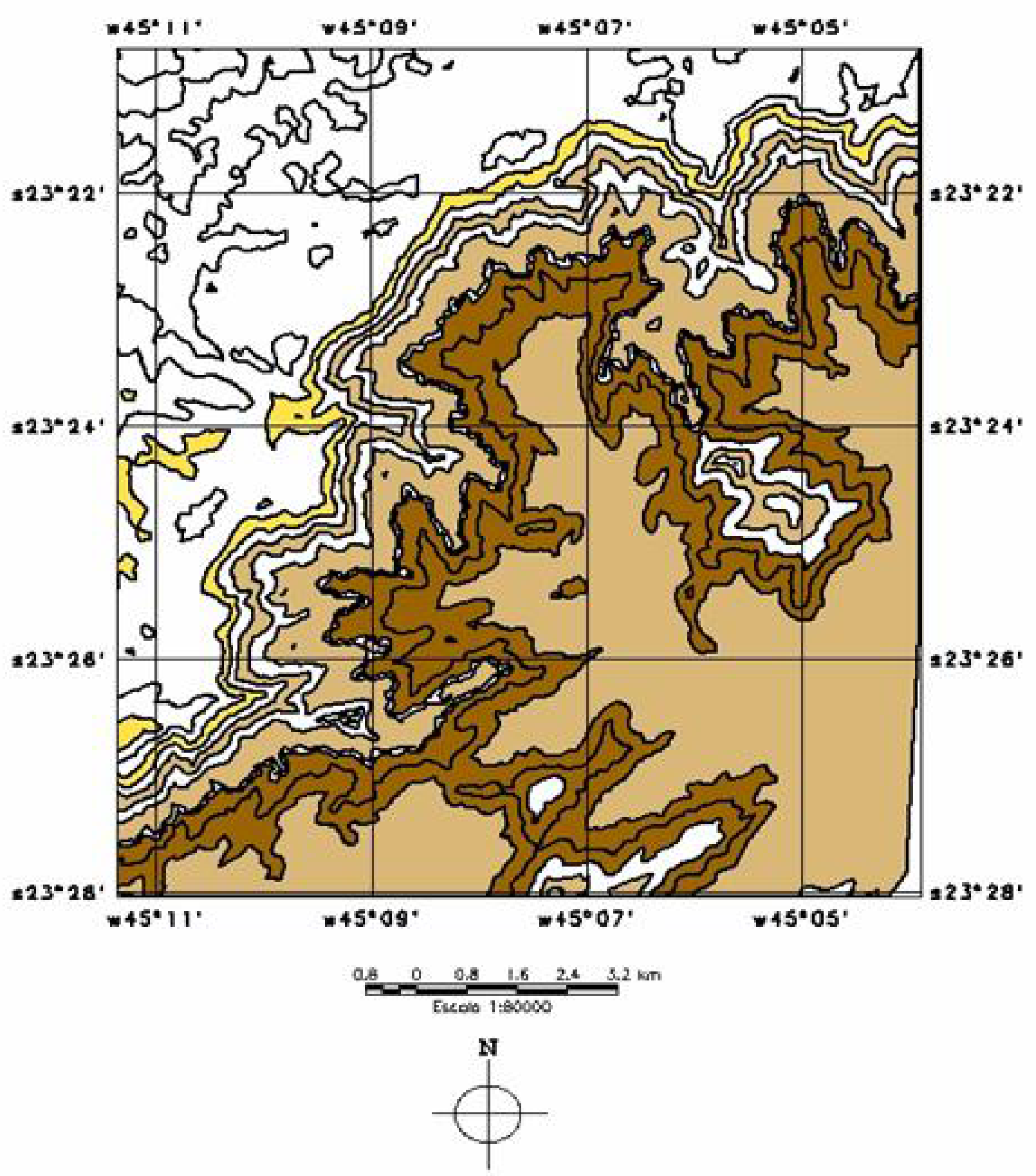

Mopo de distribuiçõo do número de espécies de palmeiras Legenda:

$\square$ não avoliado
88 espécies
6 espécies
4 espécies

Figura 8: Mapa de distribuição do número de espécies de palmeiras segundo as classes de altitudinais avaliadas Figure 8: Distribution map of the number of palms species according to class of altitude evaluated. 


\begin{tabular}{rcc}
\hline Altitude $(\mathrm{m})$ & Longitude & Latitude \\
\hline 0 & - & - \\
100 & 45W08'28”, & 23S25'30” \\
200 & 45W08'45” & 23S25'26” \\
400 & 45W09'10” & 23S25'10” \\
600 & 45W09'19” & 23S25'00” \\
50 & 45W09'33” & 23S24'47” \\
\hline
\end{tabular}

Tabela 2: Ocorrência de palmeiras no gradiente altitudinal: 0m - Restinga de Picinguaba; 100m-850m - Trilha da Vargem-Grande, município de Ubatuba/SP

Table 2: Occurrence of palm along an altitudinal gradient in Ubatuba: 0 meters - Picinguaba restinga; 100 - 850 meters - Vargem-Grande transect.

\begin{tabular}{c|c|c|c|c|c|c}
\hline \multirow{2}{*}{ ESPÉCIES } & \multicolumn{7}{|c}{ ALTITUDE } \\
\cline { 2 - 7 } & $\mathbf{0 m}$ & $\mathbf{1 0 0}$ & $\mathbf{2 0 0}$ & $\mathbf{4 0 0}$ & $\mathbf{6 0 0}$ & $\mathbf{8 5 0}$ \\
\hline Astrocaryum & $\mathrm{X}$ & $\mathrm{X}$ & $\mathrm{X}$ & $\mathrm{X}$ & $\mathrm{X}$ & \\
aculeatissimum & & $\mathrm{X}$ & $\mathrm{X}$ & $\mathrm{X}$ & $\mathrm{X}$ & $\mathrm{X}$ \\
Geonoma gamiova & & $\mathrm{X}$ & & & & $\mathrm{X}$ \\
G. pohliana & & $\mathrm{X}$ & $\mathrm{X}$ & $\mathrm{X}$ & $\mathrm{X}$ & \\
G. elegans & $\mathrm{X}$ & $\mathrm{X}$ & $\mathrm{X}$ & & & \\
Attalea dubia & & $\mathrm{X}$ & $\mathrm{X}$ & $\mathrm{X}$ & $\mathrm{X}$ & $\mathrm{X}$ \\
Euterpe edulis & $\mathrm{X}$ & $\mathrm{X}$ & $\mathrm{X}$ & $\mathrm{X}$ & $\mathrm{X}$ & $\mathrm{X}$ \\
Syagrus peseudococos & $\mathrm{X}$ & $\mathrm{X}$ & $\mathrm{X}$ & $\mathrm{X}$ & $\mathrm{X}$ & $\mathrm{X}$ \\
Bactris setosa & $\mathrm{X}$ & $\mathrm{X}$ & $\mathrm{X}$ & & & \\
B. hatschbachii & $\mathrm{X}$ & & $\mathrm{X}$ & $\mathrm{X}$ & $\mathrm{X}$ & \\
\hline Total de Espécies & $\mathbf{6}$ & $\mathbf{8}$ & $\mathbf{8}$ & $\mathbf{6}$ & $\mathbf{6}$ & $\mathbf{4}$ \\
\hline
\end{tabular}

